ESAIM: PROCEEDINGS, March 2013, Vol. 39, p. 57-65

M. Belhaq, P. Lafitte and T. Lelièvre Editors

\title{
CENTER MANIFOLD REDUCTION OF THE HOPF-HOPF BIFURCATION IN A TIME DELAY SYSTEM
}

\author{
Christoffer Heckman ${ }^{1}$, Jakob Kotas ${ }^{2}$ and Richard Rand ${ }^{3}$
}

\begin{abstract}
In this work, a differential delay equation (DDE) with a cubic nonlinearity is analyzed as two parameters are varied by means of a center manifold reduction. This reduction is applied directly to the case where the system undergoes a Hopf-Hopf bifurcation. This procedure replaces the original DDE with four first-order ODEs, an approximation valid in the neighborhood of the Hopf-Hopf bifurcation. Analysis of the resulting ODEs shows that two separate periodic motions (limit cycles) and an additional quasiperiodic motion are born out of the Hopf-Hopf bifurcation. The analytical results are shown to agree with numerical results obtained by applying the continuation software package DDE-BIFTOOL to the original DDE. This system has analogues in coupled microbubble oscillators.
\end{abstract}

\section{INTRODUCTION}

Delay in dynamical systems is exhibited whenever the system's behavior is dependent at least in part on its history. Many technological and biological systems are known to exhibit such behavior; coupled laser systems, high-speed milling, population dynamics and gene expression are some examples of delayed systems. This work analyzes a simple differential delay equation that is motivated by a system of two microbubbles coupled by acoustic forcing, previously studied by Heckman et al. [1] [2]. The propagation time of sound in the fluid gives rise to a time delay between the two bubbles. The system under study has the same linearization as the equations previously studied, but the sophisticated nonlinear interaction terms in the bubble equations have been replaced by a cubic term in order to provide first insights into the full bubble equations.

In particular, the system of coupled microbubbles has been witnessed to exhibit damped oscillation, excited oscillations (i.e. a stable limit cycle created as a result of a supercritical Hopf bifurcation), and quasiperiodic oscillations. These latter dynamics are unexplained by previous work, but it has been previously suggested that these dynamics are the result of a Neimark-Sacker bifurcation. This work explores this possibility by analyzing the dynamics of an analogous system by means of a center manifold reduction. However, in contrast with previous work, this reduction will analyze the Hopf-Hopf bifurcation that results when two parameters (corresponding to the speed of sound in the fluid and the delay propagation time) are varied.

\section{Center Manifold Reduction}

The system under analysis is motivated by the Rayleigh-Plesset Equation with Delay Coupling (RPE), as studied by Heckman et al. [1] [2]. The equation of motion for a spherical bubble contains quadratic nonlinearities

${ }^{1}$ Field of Theoretical \& Applied Mechanics ; Cornell University; Ithaca, NY USA

2 Department of Applied Mathematics; University of Washington; Seattle, WA USA

${ }^{3}$ Department of Mathematics and Department of Mechanical \& Aerospace Engineering; Cornell University; Ithaca, NY USA

(C) EDP Sciences, SMAI 2013 
and multiple parameters quantifying the fluids' mechanical properties; the equations studied in this work are designed to capture salient dynamical properties while simplifying analysis. The system is :

$$
\kappa \ddot{x}+4 \dot{x}+4 \kappa x+10 \dot{x}(t-T)=\epsilon x^{3} .
$$

Eq. (1) has the same linearization as the RPE, with a cubic nonlinear term added to it. This system has an equilibrium point at $x=0$ that will correspond to the local behavior of the RPE's equilibrium point as a result.

In order to put Eq. (1) into a form amenable to treatment by functional analysis, we draw on the method used by Kalmár-Nagy et al. [3] and Rand [4], [5]. The operator differential equation for this system will now be developed. Eq. (1) may be written in the form :

$$
\dot{\mathbf{x}}(t)=\mathbf{L}(\kappa) \mathbf{x}(t)+\mathbf{R}(\kappa) \mathbf{x}(t-\tau)+\mathbf{f}(\mathbf{x}(t), \mathbf{x}(t-\tau), \kappa)
$$

where

$$
\begin{gathered}
\mathbf{x}(t)=\left(\begin{array}{l}
x(t) \\
\dot{x}(t)
\end{array}\right)=\left(\begin{array}{l}
x_{1} \\
x_{2}
\end{array}\right) \\
\mathbf{L}(\kappa)=\left(\begin{array}{cc}
0 & 1 \\
-4 & -4 / \kappa
\end{array}\right), \mathbf{R}(\kappa)=\left(\begin{array}{cc}
0 & 0 \\
0 & -10 / \kappa
\end{array}\right)
\end{gathered}
$$

and

$$
\mathbf{f}(\mathbf{x}(t), \mathbf{x}(t-\tau), \kappa)=\left(\begin{array}{c}
0 \\
(\epsilon / \kappa) x_{1}^{3}
\end{array}\right)
$$

Note that the initial conditions to a differential delay equation consists of a function defined on $-\tau \leq t \leq 0$. As $t$ increases from zero, the initial function on $[-\tau, 0]$ evolves to one on $[-\tau+t, t]$. This implies the flow is determined by a function whose initial conditions are shifting. In order to make the differential delay equation problem tenable to analysis, it is advantageous to recast it in the context of functional analysis.

To accomplish this, we consider a function space of continuously differential functions on $[-\tau, 0]$. The time variable $t$ specifies which function is being considered, namely the one corresponding to the interval $[-\tau+t, t]$. The phase variable $\theta$ specifies a point in the interval $[-\tau, 0]$.

Now, the variable $\mathbf{x}(t+\theta)$ represents the point in the function space which has evolved from the initial condition function $\mathbf{x}(\theta)$ at time $t$. From the point of view of the function space, $t$ is now a parameter, whereas $\theta$ is the independent variable. To emphasize this new definition, we write

$$
\mathbf{x}_{t}(\theta)=\mathbf{x}(t+\theta), \quad \theta \in[-\tau, 0] .
$$

The delay differential equation may therefore be expressed as

$$
\dot{\mathbf{x}}_{t}=\mathcal{A} \mathbf{x}_{t}+\mathcal{F}\left(\mathbf{x}_{t}\right),
$$

The linear mapping of the original equation is given by

$$
\mathcal{L}(\phi(\theta))=\mathbf{L}(\kappa) \phi(0)+\mathbf{R}(\kappa) \phi(-\tau)
$$

where $\mathbf{x}(t)=\phi(t)$ for $t \in[-\tau, 0], \mathbf{F}: \mathcal{H} \rightarrow \mathbb{R}^{2}$ is a nonlinear functional defined by

$$
\mathbf{F}(\phi(\theta))=\mathbf{f}(\phi(0), \phi(-\tau)),
$$

and where $\mathcal{H}=C\left([-\tau, 0], \mathbb{R}^{2}\right)$ is the Banach space of continuously differentiable functions $\mathbf{u}=\left(\begin{array}{l}u_{1} \\ u_{2}\end{array}\right)$ from $[-\tau, 0]$ into $\mathbb{R}^{2}$.

If $\kappa^{*}$ is the critical value of the bifurcation parameter, and noting that $\frac{\partial \mathbf{x}_{t}(\theta)}{\partial t}=\frac{\partial \mathbf{x}_{t}(\theta)}{\partial \theta}$ (which follows from $\left.\mathbf{x}_{t}(\theta)=\mathbf{x}(t+\theta)\right)$, then when $\kappa=\kappa^{*}$ the operator differential equation has components 
and

$$
\mathcal{A} \mathbf{u}(\theta)= \begin{cases}\frac{d}{d \theta} \mathbf{u}(\theta) & \theta \in[-\tau, 0) \\ \mathbf{L u}(0)+\mathbf{R u}(-\tau) & \theta=0\end{cases}
$$

$$
\mathcal{F}(\mathbf{u}(\theta))= \begin{cases}\mathbf{0} & \theta \in[-\tau, 0) \\
\left(\begin{array}{c}
0 \\
(\epsilon / \kappa) u_{1}(0)^{3}
\end{array}\right) & \theta=0\end{cases}
$$

Eqs. (3) and (4) are representations of eq. (1) in "canonical form." They contain the corresponding linear and nonlinear parts of eq. (1) as the boundary conditions to the full evolution equation (2).

A stability analysis of eq. (3) alone provides insight into the asymptotic stability of the original equations. In the case when eq. (3) has neutral stability (i.e. has eigenvalues with real part zero), analysis of eq. (4) is necessary. The purpose of the center manifold reduction is to project the dynamics of the infinite-dimensional singular case onto a low-dimensional subspace on which the dynamics are more analytically tractable.

At a bifurcation, the critical eigenvalues of the operator $\mathcal{A}$ coincide with the critical roots of the characteristic equation. In this system, the target of analysis is a Hopf-Hopf bifurcation, a codimension-2 bifurcation that has a four-dimensional center manifold [6]. Consequently, there will be two pairs of critical eigenvalues $\pm i \omega_{a}$ and $\pm i \omega_{b}$ with real part zero. Each eigenvalue has an eigenspace spanned by the real and imaginary parts of its corresponding complex eigenfunction. These eigenfunctions are denoted $\mathbf{s}_{a}(\theta), \mathbf{s}_{b}(\theta) \in \mathcal{H}$.

The eigenfunctions satisfy

$$
\begin{aligned}
& \mathcal{A} \mathbf{s}_{a}(\theta)=i \omega_{a} \mathbf{s}_{a}(\theta) \\
& \mathcal{A} \mathbf{s}_{b}(\theta)=i \omega_{b} \mathbf{s}_{b}(\theta) ;
\end{aligned}
$$

or equivalently,

$$
\begin{aligned}
& \mathcal{A}\left(\mathbf{s}_{a 1}(\theta)+i \mathbf{s}_{a 2}(\theta)\right)=i \omega_{a}\left(\mathbf{s}_{a 1}(\theta)+i \mathbf{s}_{a 2}(\theta)\right) \\
& \mathcal{A}\left(\mathbf{s}_{b 1}(\theta)+i \mathbf{s}_{b 2}(\theta)\right)=i \omega_{b}\left(\mathbf{s}_{b 1}(\theta)+i \mathbf{s}_{b 2}(\theta)\right)
\end{aligned}
$$

Equating real and imaginary parts in eq. (5) and eq. (6) gives

$$
\begin{aligned}
& \mathcal{A} \mathbf{s}_{a 1}(\theta)=-\omega_{a} \mathbf{s}_{a 2}(\theta) \\
& \mathcal{A} \mathbf{s}_{a 2}(\theta)=\omega_{a} \mathbf{s}_{a 1}(\theta) \\
& \mathcal{A} \mathbf{s}_{b 1}(\theta)=-\omega_{b} \mathbf{s}_{b 2}(\theta) \\
& \mathcal{A} \mathbf{s}_{b 2}(\theta)=\omega_{b} \mathbf{s}_{b 1}(\theta) .
\end{aligned}
$$

Applying the definition of $\mathcal{A}$ to eqs. (7)- (10) produces the differential equations

$$
\begin{aligned}
\frac{d}{d \theta} \mathbf{s}_{a 1}(\theta) & =-\omega_{a} \mathbf{s}_{a 2}(\theta) \\
\frac{d}{d \theta} \mathbf{s}_{a 2}(\theta) & =\omega_{a} \mathbf{s}_{a 1}(\theta) \\
\frac{d}{d \theta} \mathbf{s}_{b 1}(\theta) & =-\omega_{b} \mathbf{s}_{b 2}(\theta) \\
\frac{d}{d \theta} \mathbf{s}_{b 1}(\theta) & =\omega_{b} \mathbf{s}_{b 1}(\theta)
\end{aligned}
$$


with boundary conditions

$$
\begin{aligned}
\mathbf{L s}_{a 1}(0)+\mathbf{R s}_{a 1}(-\tau) & =-\omega_{a} \mathbf{s}_{a 2}(0) \\
\mathbf{L s}_{a 2}(0)+\mathbf{R s}_{a 2}(-\tau) & =\omega_{a} \mathbf{s}_{a 1}(0) \\
\mathbf{L s}_{b 1}(0)+\mathbf{R s}_{b 1}(-\tau) & =-\omega_{b} \mathbf{s}_{b 2}(0) \\
\mathbf{L s}_{b 2}(0)+\mathbf{R s}_{b 2}(-\tau) & =\omega_{b} \mathbf{s}_{b 1}(0)
\end{aligned}
$$

The general solution to the differential equations (11)-(14) is :

$$
\begin{aligned}
& \mathbf{s}_{a 1}(\theta)=\cos \left(\omega_{a} \theta\right) \mathbf{c}_{a 1}-\sin \left(\omega_{a} \theta\right) \mathbf{c}_{a 2} \\
& \mathbf{s}_{a 2}(\theta)=\sin \left(\omega_{a} \theta\right) \mathbf{c}_{a 1}+\cos \left(\omega_{a} \theta\right) \mathbf{c}_{a 2} \\
& \mathbf{s}_{b 1}(\theta)=\cos \left(\omega_{b} \theta\right) \mathbf{c}_{b 1}-\sin \left(\omega_{b} \theta\right) \mathbf{c}_{b 2} \\
& \mathbf{s}_{b 2}(\theta)=\sin \left(\omega_{b} \theta\right) \mathbf{c}_{b 2}+\cos \left(\omega_{b} \theta\right) \mathbf{c}_{b 2}
\end{aligned}
$$

where $\mathbf{c}_{\alpha i}=\left(\begin{array}{l}c_{\alpha i 1} \\ c_{\alpha i 2}\end{array}\right)$. This results in eight unknowns which may be solved by applying the boundary conditions (15)-(18). However, since we are searching for a nontrivial solution to these equations, they must be linearly dependent. We set the value of four of the unknowns to simplify the final result :

$$
c_{a 11}=1, \quad c_{a 21}=0, \quad c_{b 11}=1, \quad c_{b 21}=0 .
$$

This allows eqs. (15)-(18) to be solved uniquely, yielding

$$
\mathbf{c}_{a 1}=\left(\begin{array}{l}
1 \\
0
\end{array}\right), \quad \mathbf{c}_{a 2}=\left(\begin{array}{c}
0 \\
\omega_{a}
\end{array}\right), \quad \mathbf{c}_{b 1}=\left(\begin{array}{l}
1 \\
0
\end{array}\right), \quad \mathbf{c}_{b 2}=\left(\begin{array}{c}
0 \\
\omega_{b}
\end{array}\right)
$$

Next, the vectors that span the dual space $\mathcal{H}^{*}$ must be calculated. The boundary value problem associated with this case has the same differential equations (11)-(14) except on $\mathbf{n}_{\alpha i}$ rather than on $\mathbf{s}_{\alpha i}$; in place of boundary conditions (15)-(18), there are boundary conditions

$$
\begin{aligned}
\mathbf{L}^{T} \mathbf{n}_{a 1}(0)+\mathbf{R}^{T} \mathbf{n}_{a 1}(\tau) & =\omega_{a} \mathbf{n}_{a 2}(0) \\
\mathbf{L}^{T} \mathbf{n}_{a 2}(0)+\mathbf{R}^{T} \mathbf{n}_{a 2}(\tau) & =-\omega_{a} \mathbf{n}_{a 1}(0) \\
\mathbf{L}^{T} \mathbf{n}_{b 1}(0)+\mathbf{R}^{T} \mathbf{n}_{b 1}(\tau) & =\omega_{b} \mathbf{n}_{b 2}(0) \\
\mathbf{L}^{T} \mathbf{n}_{b 2}(0)+\mathbf{R}^{T} \mathbf{s}_{b 2}(\tau) & =-\omega_{b} \mathbf{n}_{b 1}(0)
\end{aligned}
$$

The general solution to the differential equation associated with this boundary value problem is

$$
\begin{aligned}
& \mathbf{n}_{a 1}(\sigma)=\cos \left(\omega_{a} \sigma\right) \mathbf{d}_{a 1}-\sin \left(\omega_{a} \sigma\right) \mathbf{d}_{a 2} \\
& \mathbf{n}_{a 2}(\sigma)=\sin \left(\omega_{a} \sigma\right) \mathbf{d}_{a 1}+\cos \left(\omega_{a} \sigma\right) \mathbf{d}_{a 2} \\
& \mathbf{n}_{b 1}(\sigma)=\cos \left(\omega_{b} \sigma\right) \mathbf{d}_{b 1}-\sin \left(\omega_{b} \sigma\right) \mathbf{d}_{b 2} \\
& \mathbf{n}_{b 2}(\sigma)=\sin \left(\omega_{b} \sigma\right) \mathbf{d}_{b 2}+\cos \left(\omega_{b} \sigma\right) \mathbf{d}_{b 2}
\end{aligned}
$$

Again, these equations are not linearly independent. Four more equations may be generated by orthonormalizing the $\mathbf{n}_{\alpha i}$ and $\mathbf{s}_{\alpha j}$ vectors (conditions on the bilinear form between these vectors) : 


$$
\begin{aligned}
\left(\mathbf{n}_{a 1}, \mathbf{s}_{a 1}\right)=1, & \left(\mathbf{n}_{a 1}, \mathbf{s}_{a 2}\right)=0 \\
\left(\mathbf{n}_{b 1}, \mathbf{s}_{b 1}\right)=1, & \left(\mathbf{n}_{b 1}, \mathbf{s}_{b 2}\right)=0
\end{aligned}
$$

where the bilinear form employed is $(\mathbf{v}, \mathbf{u})=\mathbf{v}^{T}(0) \mathbf{u}(0)+\int_{-\tau}^{0} \mathbf{v}^{T}(\xi+\tau) \mathbf{R u}(\xi) \mathrm{d} \xi$.

Eqs. (11)-(14) combined with (20)-(25) may be solved uniquely for $d_{\alpha i j}$ in terms of the system parameters. Using eqs. (19) as the values for $\mathbf{c}_{\alpha i}$ and substituting relevant values of the parameters $\kappa^{*}=6.8916, \tau^{*}=2.9811$, $\omega_{a}=1.4427$, and $\omega_{b}=2.7726$ to center the perturbation method at the Hopf-Hopf bifurcation yields

$$
\begin{aligned}
& \mathbf{d}_{a 1}=\left(\begin{array}{c}
0.4786 \\
0.1471
\end{array}\right), \quad \mathbf{d}_{a 2}=\left(\begin{array}{c}
-0.4079 \\
0.1726
\end{array}\right), \\
& \mathbf{d}_{b 1}=\left(\begin{array}{c}
0.1287 \\
-0.1088
\end{array}\right), \quad \mathbf{d}_{b 2}=\left(\begin{array}{c}
0.1570 \\
0.0892
\end{array}\right)
\end{aligned}
$$

\section{Flow on the Center Manifold}

The solution vector $\mathbf{x}_{t}(\theta)$ may be understood as follows. The center subspace is four-dimensional and spanned by the vectors $\mathbf{s}_{\alpha i}$. The solution vector is decomposed into four components $y_{\alpha i}$ in the $\mathbf{s}_{\alpha i}$ basis, but it also contains a part that is out of the center subspace. This component is infinite-dimensional, and is captured by the term $\mathbf{w}=\left(\begin{array}{l}w_{1} \\ w_{2}\end{array}\right)$ transverse to the center subspace. The solution vector may therefore be written as

$$
\mathbf{x}_{t}(\theta)=y_{a 1}(t) \mathbf{s}_{a 1}(\theta)+y_{a 2}(t) \mathbf{s}_{a 2}(\theta)+y_{b 1}(t) \mathbf{s}_{b 1}(\theta)+y_{b 2}(t) \mathbf{s}_{b 2}(\theta)+\mathbf{w}(t)(\theta)
$$

Note that, by definition,

$$
\begin{aligned}
& y_{a 1}(t)=\left.\left(\mathbf{n}_{a 1}, \mathbf{x}_{t}\right)\right|_{\theta=0} \\
& y_{a 2}(t)=\left.\left(\mathbf{n}_{a 2}, \mathbf{x}_{t}\right)\right|_{\theta=0} \\
& y_{b 1}(t)=\left.\left(\mathbf{n}_{b 1}, \mathbf{x}_{t}\right)\right|_{\theta=0} \\
& y_{b 2}(t)=\left.\left(\mathbf{n}_{b 2}, \mathbf{x}_{t}\right)\right|_{\theta=0}
\end{aligned}
$$

The nonlinear part of the operator is crucial for transforming the operator differential equation into the canonical form described by Guckenheimer \& Holmes. This nonlinear operator is

$$
\begin{aligned}
& \mathcal{F}\left(\mathbf{x}_{t}\right)(\theta)=\mathcal{F}\left(y_{a 1}(t) \mathbf{s}_{a 1}+y_{a 2}(t) \mathbf{s}_{a 2}+y_{b 1}(t) \mathbf{s}_{b 1}+y_{b 2}(t) \mathbf{s}_{b 2}+\mathbf{w}(t)\right)(\theta) \\
& =\left\{\begin{array}{cl}
\mathbf{0} & \theta \in[-\tau, 0) \\
\left(\begin{array}{cl}
0 \\
\left(\frac{\epsilon}{\kappa}\left(y_{a 1} c_{a 11}+y_{a 2} c_{a 21}+y_{b 1} c_{b 11}+y_{b 2} c_{b 21}+w_{1}(t)(0)\right)^{3}\right.
\end{array}\right) & \theta=0
\end{array}\right.
\end{aligned}
$$

In order to derive the canonical form, we take $\frac{d}{d t}$ of $y_{\alpha i}(t)$ from eqs. (26)-(29) and carry through the differentiation to the factors of the bilinear form. Noting that $\frac{d}{d t} \mathbf{n}_{\alpha i}=0$, 


$$
\begin{aligned}
\dot{y}_{\alpha 1} & =\left.\left(\mathbf{n}_{\alpha 1}, \dot{\mathbf{x}}_{t}\right)\right|_{\theta=0}=\left.\left(\mathbf{n}_{\alpha 1}, \mathcal{A} \mathbf{x}_{t}+\mathcal{F}\left(\mathbf{x}_{t}\right)\right)\right|_{\theta=0} \\
& =\left.\left(\mathbf{n}_{\alpha 1}, \mathcal{A} \mathbf{x}_{t}\right)\right|_{\theta=0}+\left.\left(\mathbf{n}_{\alpha 1}, \mathcal{F}\left(\mathbf{x}_{t}\right)\right)\right|_{\theta=0} \\
& =\left.\left(\mathcal{A}^{*} \mathbf{n}_{\alpha 1}, \mathbf{x}_{t}\right)\right|_{\theta=0}+\left.\left(\mathbf{n}_{\alpha 1}, \mathcal{F}\left(\mathbf{x}_{t}\right)\right)\right|_{\theta=0} \\
& =\left.\omega_{\alpha}\left(\mathbf{n}_{\alpha 2}, \mathbf{x}_{t}\right)\right|_{\theta=0}+\left.\left(\mathbf{n}_{\alpha 1}, \mathcal{F}\left(\mathbf{x}_{t}\right)\right)\right|_{\theta=0} \\
& =\omega_{\alpha} y_{\alpha 2}+\mathbf{n}_{\alpha 1}^{T}(0) \mathbf{F}
\end{aligned}
$$

and similarly,

$$
\dot{y}_{\alpha 2}=-\omega_{\alpha} y_{\alpha 1}+\mathbf{n}_{\alpha 2}^{T}(0) \mathbf{F}
$$

where $\mathbf{F}=\mathcal{F}\left(\mathbf{x}_{t}\right)(0)=\mathcal{F}\left(y_{a 1}(t) \mathbf{s}_{a 1}(0)+y_{a 2}(t) \mathbf{s}_{a 2}(0)+y_{b 1}(t) \mathbf{s}_{b 1}(0)+y_{b 2}(t) \mathbf{s}_{b 2}(0)+\mathbf{w}(t)(0)\right)$, recalling that $\mathcal{F}=\mathcal{F}(\theta)$, and this notation corresponds to setting $\theta=0$. Substituting in the definition of $\mathbf{n}_{\alpha i}$ and $\mathbf{F}$,

$$
\begin{aligned}
& \dot{y}_{a 1}=\omega_{a} y_{a 2}+\frac{\epsilon d_{a 12}\left(y_{a 1}+y_{b 1}+w_{1}\right)^{3}}{\kappa} \\
& \dot{y}_{a 2}=-\omega_{a} y_{a 1}+\frac{\epsilon d_{a 22}\left(y_{a 1}+y_{b 1}+w_{1}\right)^{3}}{\kappa} \\
& \dot{y}_{b 1}=\omega_{b} y_{a 2}+\frac{\epsilon d_{b 12}\left(y_{a 1}+y_{b 1}+w_{1}\right)^{3}}{\kappa} \\
& \dot{y}_{b 2}=-\omega_{b} y_{b 1}+\frac{\epsilon d_{b 22}\left(y_{a 1}+y_{b 1}+w_{1}\right)^{3}}{\kappa}
\end{aligned}
$$

where we have used eq. (19). Recall that the center manifold is tangent to the four-dimensional $y_{\alpha i}$ center subspace at the origin and $\mathbf{w}$ may be approximated by a quadratic in $y_{\alpha i}$. Therefore, the terms $w_{1}$ in eqs. (30)(33) may be neglected, as their contribution is greater than third order, which had previously been neglected. To analyze this eqs. (30)-(33), a van der Pol transformation is applied :

$$
\begin{aligned}
& y_{a 1}(t)=r_{a}(t) \cos \left(\omega_{a} t+\theta_{a}(t)\right) \\
& y_{a 2}(t)=-r_{a}(t) \sin \left(\omega_{a} t+\theta_{a}(t)\right) \\
& y_{b 1}(t)=r_{b}(t) \cos \left(\omega_{b} t+\theta_{b}(t)\right) \\
& y_{b 2}(t)=-r_{b}(t) \sin \left(\omega_{b} t+\theta_{b}(t)\right)
\end{aligned}
$$

transforms the coupled differential equations (30)-(33) into

$$
\begin{aligned}
& \dot{r}_{a}=\frac{\epsilon}{\kappa}\left(\cos \left(t \omega_{a}+\theta_{a}\right) r_{a}+\cos \left(t \omega_{b}+\theta_{b}\right) r_{b}\right)^{3}\left(d_{a 12} \cos \left(t \omega_{a}+\theta_{a}\right)-d_{a 22} \sin \left(t \omega_{a}+\theta_{a}\right)\right) \\
& \dot{\theta}_{a}=\frac{-\epsilon}{\kappa r_{a}}\left(\cos \left(t \omega_{a}+\theta_{a}\right) r_{a}+\cos \left(t \omega_{b}+\theta_{b}\right) r_{b}\right)^{3}\left(d_{a 22} \cos \left(t \omega_{a}+\theta_{a}\right)+d_{a 12} \sin \left(t \omega_{a}+\theta_{a}\right)\right) \\
& \dot{r}_{b}=\frac{\epsilon}{\kappa}\left(\cos \left(t \omega_{a}+\theta_{a}\right) r_{a}+\cos \left(t \omega_{b}+\theta_{b}\right) r_{b}\right)^{3}\left(d_{b 12} \cos \left(t \omega_{b}+\theta_{b}\right)-d_{b 22} \sin \left(t \omega_{b}+\theta_{b}\right)\right) \\
& \dot{\theta}_{b}=\frac{-\epsilon}{\kappa r_{b}}\left(\cos \left(t \omega_{a}+\theta_{a}\right) r_{a}+\cos \left(t \omega_{b}+\theta_{b}\right) r_{b}\right)^{3}\left(d_{b 22} \cos \left(t \omega_{b}+\theta_{b}\right)+d_{b 12} \sin \left(t \omega_{b}+\theta_{b}\right)\right)
\end{aligned}
$$


By averaging the differential equations (34)-(37) over a single period of $t \omega_{\alpha}+\theta_{\alpha}$, the $\theta_{\alpha}$ dependence of the $\dot{r}_{\alpha}$ equations may be eliminated. Note that $\omega_{a}$ and $\omega_{b}$ are non-resonant frequencies, so averages may be taken independently of one another.

$$
\begin{aligned}
& \frac{\omega_{a}}{2 \pi} \int_{\theta_{a}}^{\theta_{a}+\frac{2 \pi}{\omega_{a}}} \dot{r}_{a} \mathrm{~d} t=\frac{3}{8} \frac{\epsilon}{\kappa} d_{a 12} r_{a}\left(2 r_{b}^{2}+r_{a}^{2}\right) \\
& \frac{\omega_{b}}{2 \pi} \int_{\theta_{b}}^{\theta_{b}+\frac{2 \pi}{\omega_{b}}} \dot{r}_{b} \mathrm{~d} t=\frac{3}{8} \frac{\epsilon}{\kappa} d_{b 12} r_{b}\left(2 r_{a}^{2}+r_{b}^{2}\right)
\end{aligned}
$$

According to Guckenheimer \& Holmes, the normal form for a Hopf-Hopf bifurcation in polar coordinates is

$$
\begin{aligned}
\frac{\mathrm{d} r_{a}(t)}{\mathrm{d} t} & =\mu_{a} r_{a}+a_{11} r_{a}^{3}+a_{12} r_{a} r_{b}^{2}+\mathcal{O}\left(|r|^{5}\right) \\
\frac{\mathrm{d} r_{b}(t)}{\mathrm{d} t} & =\mu_{b} r_{b}+a_{22} r_{b}^{3}+a_{21} r_{b} r_{a}^{2}+\mathcal{O}\left(|r|^{5}\right) \\
\frac{\mathrm{d} \theta_{a}(t)}{\mathrm{d} t} & =\omega_{a}+\mathcal{O}\left(|r|^{2}\right) \\
\frac{\mathrm{d} \theta_{b}(t)}{\mathrm{d} t} & =\omega_{b}+\mathcal{O}\left(|r|^{2}\right)
\end{aligned}
$$

where $\mu_{i}=\Re \frac{\mathrm{d} \lambda_{i}\left(\tau^{*}\right)}{\mathrm{d} \tau}$, and $\tau^{*}$ is the critical time-delay for the Hopf-Hopf bifurcation (note that this bifurcation is of codimension 2, so both $\tau=\tau^{*}$ and $\kappa=\kappa^{*}$ at the bifurcation). Taking the derivative of the characteristic equation with respect to $\tau$ and solving for $\frac{\mathrm{d} \lambda(\tau)}{\mathrm{d} \tau}$ gives

$$
\frac{\mathrm{d} \lambda(\tau)}{\mathrm{d} \tau}=\frac{5 \lambda(\tau)^{2}}{5+2 \exp (\tau \lambda(\tau))-5 \tau \lambda(\tau)+\exp (\tau \lambda(\tau)) \kappa \lambda(\tau)}
$$

Letting $\lambda(\tau)=i \omega_{\alpha}(\tau)$ and substituting in $\tau=\tau^{*}, \kappa=\kappa^{*}$, as well as $\omega_{a}$ and $\omega_{b}$ respectively yields

$$
\begin{aligned}
\mu_{a} & =-0.1500 \Delta \\
\mu_{b} & =0.2133 \Delta
\end{aligned}
$$

where $\Delta=\tau-\tau^{*}$. This results in the equations for the flow on the center manifold :

$$
\begin{aligned}
& \dot{r}_{a}=-0.1500 \Delta r_{a}+0.0080 r_{a}\left(2 r_{b}^{2}+r_{a}^{2}\right) \\
& \dot{r}_{b}=0.2133 \Delta r_{b}-0.0059 r_{b}\left(2 r_{a}^{2}+r_{b}^{2}\right)
\end{aligned}
$$

To normalize the coefficients and finally obtain the flow on the center manifold in normal form, let $\bar{r}_{a}=$ $r_{a} \sqrt{0.0080}$ and $\bar{r}_{b}=r_{b} \sqrt{0.0059}$, resulting in :

$$
\begin{aligned}
\dot{\bar{r}}_{a} & =-0.1500 \Delta \bar{r}_{a}+\bar{r}_{a}^{3}+2.7042 \bar{r}_{a} \bar{r}_{b}^{2} \\
\dot{\bar{r}}_{b} & =0.2133 \Delta \bar{r}_{b}-1.4792 \bar{r}_{a}^{2} \bar{r}_{b}-\bar{r}_{b}^{3}
\end{aligned}
$$

This has quantities $a_{11}=1, a_{22}=-1, a_{12}=2.7042$, and $a_{21}=-1.4792$, which implies that this Hopf-Hopf bifurcation has the unfolding illustrated in Figure 1. 


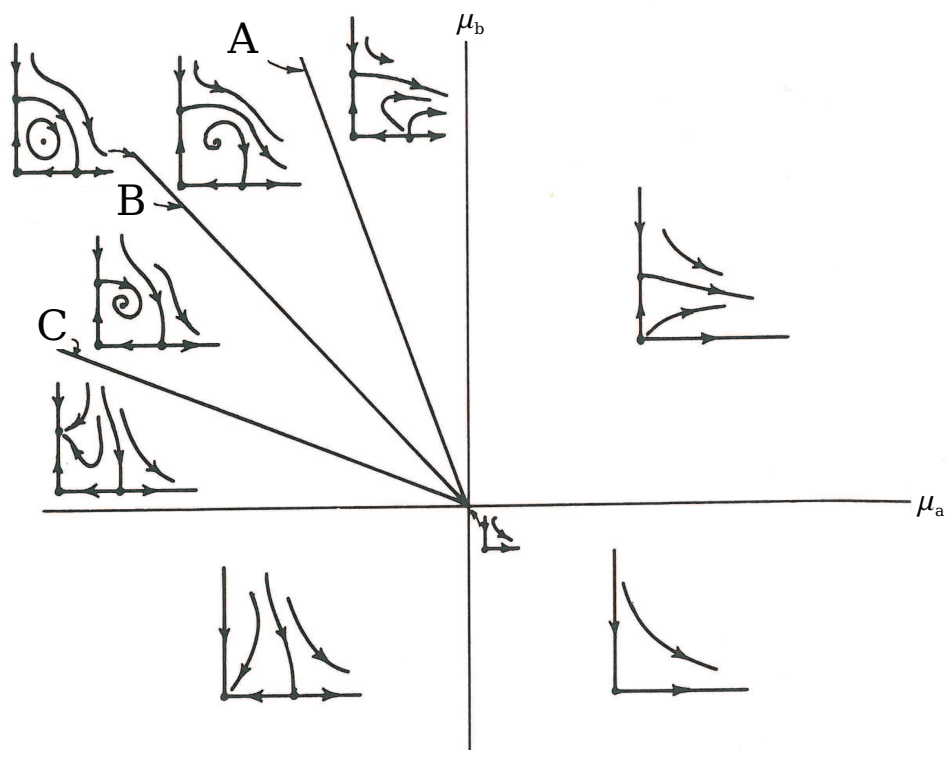

Figure 1. Partial bifurcation set and phase portraits for the unfolding of this Hopf-Hopf bifurcation. Figure reproduced from Guckenheimer \& Holmes [6] Figure 7.5.5. Note that the labels A : $\mu_{b}=a_{21} \mu_{a}, \mathrm{~B}: \mu_{b}=\mu_{a}\left(a_{21}-1\right) /\left(a_{12}+1\right), \mathrm{C}: \mu_{b}=-\mu_{a} / a_{12}$.

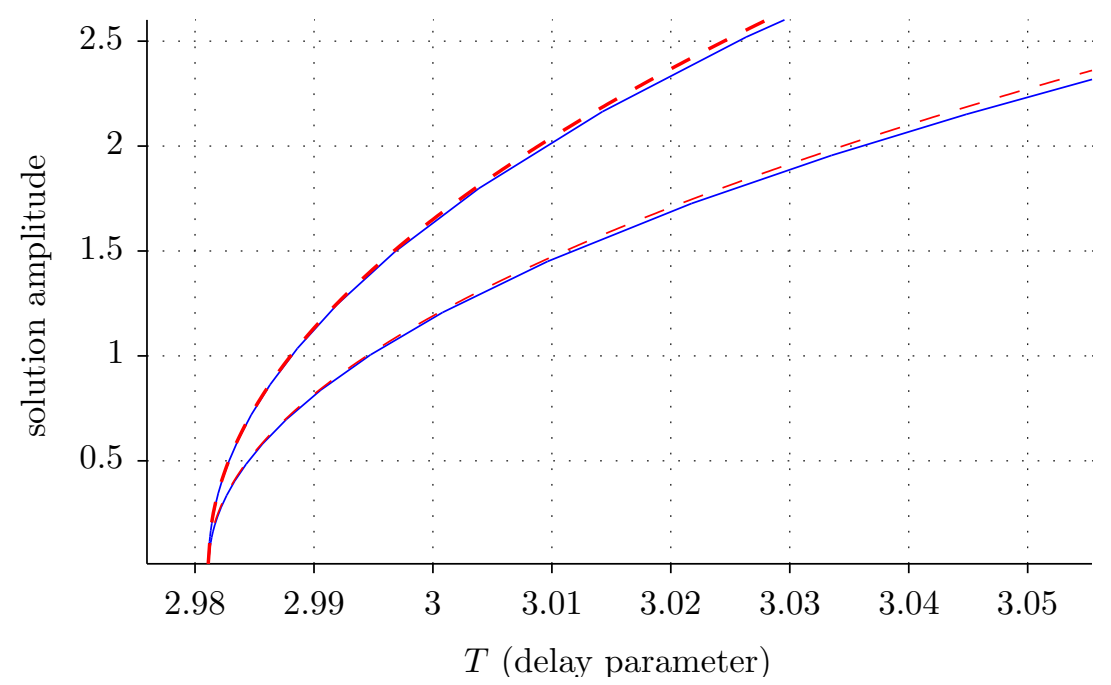

Figure 2. Comparison of predictions for the amplitudes of limit cycles bifurcating from the Hopf-Hopf point in eq. (1) obtained by (a) numerical continuation of eq. (1) using the software DDE-BIFTOOL (blue solid lines) and (b) center manifold reduction, eqs. (42), (43) (red dashed lines).

For the calculated $a_{i j}$, the bifurcation curves in Figure 1 become A : $\mu_{b}=-1.4792 \mu_{a}$, B : $\mu_{b}=-.6992 \mu_{a}$, and $\mathrm{C}: \mu_{b}=-.3697 \mu_{a}$. From eqs. (38)-(39), system (1) has $\mu_{b}=-1.422 \mu_{a}$ for the given parameter values. 
Comparison with Figure 1 shows that this implies the system exhibits two unstable limit cycles and an unstable quasiperiodic motion when $\Delta>0$. We note that the center manifold analysis is local and is expected to be valid only in the neighborhood of the origin.

For comparison, the center manifold reduction eqs. (40), (41) predict three solutions bifurcating from the Hopf-Hopf (the trivial solution notwithstanding) :

$$
\left(r_{a}, r_{b}\right)=\left\{\begin{array}{l}
(\sqrt{18.7446 \Delta}, 0) \\
(0, \sqrt{36.0238 \Delta}) \\
(\sqrt{17.7649 \Delta}, \sqrt{0.4899 \Delta}) \text { (quasiperiodic motion) }
\end{array}\right.
$$

Figure 2 shows a plot of these results along with those obtained from numerical continuation of the original system (1) with the software package DDE-BIFTOOL [7]. The two methods are shown to agree. Note that only the two limit cycles are plotted for comparison.

\section{Conclusion}

This work explored the center manifold reduction of a Hopf-Hopf bifurcation in a nonlinear differential delay equation. When analyzing a system of coupled oscillators that separately undergo Hopf bifurcation, there exists the possibility of the full system to undergo this codimension 2 bifurcation. In doing so, a wealth of sophisticated dynamics may arise that are not immediately anticipated, for instance the quasiperiodic motions. This work has served to rigorously show that a system inspired by the physical application of delay-coupled microbubble oscillators exhibits quasiperiodic motions because in part of the occurrence of a Hopf-Hopf bifurcation.

\section{REFERENCES}

[1] Heckman C.R., Sah S.M. and Rand R.H., "Dynamics of microbubble oscillators with delay coupling" Commun Nonlinear Sci Numer Simulat 15, (2010) 2735-2743.

[2] Heckman C.R. and Rand R.H., "Dynamics of Coupled Microbubbles with Large Fluid Compressibility Delays," Proc. EUROMECH 2011 Euro. Nonlin. Osc. Conf.

[3] Kalmár-Nagy T., Stépán G. and Moon F.C., "Subcritical Hopf Bifurcation in the Delay Equation Model for Machine Tool Vibrations," Nonlinear Dynamics 26, (2001) 121-142.

[4] Rand R.H., Lecture Notes in Nonlinear Vibrations, version 52 (2005),

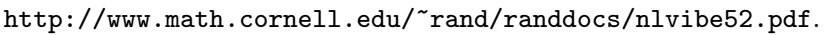

[5] Rand R.H., "Differential-Delay Equations," in Luo, A.C.J. and Sun, J-Q. (eds.), Complex Systems : Fractionality, Time-delay and Synchronization (pp. 83-117), Springer (2011).

[6] Guckenheimer J. and Holmes P., Nonlinear Oscillations, Dynamical Systems, and Bifurcations of Vector Fields (Springer, 1983)

[7] Engelborghs K., Luzyanina T., Samaey G., Roose D. and Verheyden K., DDE-BIFTOOL, available from http://twr.cs.kuleuven.be/research/software/delay/ddebiftool.shtml. 